Revue d'histoire de l'Amérique française

REVUE D.HISTOIRE DE L'AMÉRIQUE FRANÇAISE

\title{
Le mariage sous le régime français (suite)
}

\section{Paul-André Leclerc}

Volume 13, numéro 3, décembre 1959

URI : https://id.erudit.org/iderudit/301988ar

DOI : https://doi.org/10.7202/301988ar

Aller au sommaire du numéro

Éditeur(s)

Institut d'histoire de l'Amérique française

ISSN

0035-2357 (imprimé)

1492-1383 (numérique)

Découvrir la revue

Citer cet article

Leclerc, P.-A. (1959). Le mariage sous le régime français (suite). Revue d'histoire de l'Amérique française, 13(3), 374-401. https://doi.org/10.7202/301988ar d'utilisation que vous pouvez consulter en ligne.

https://apropos.erudit.org/fr/usagers/politique-dutilisation/ 


\title{
LE MARIAGE SOUS LE RÉGIME FRANÇAIS *
}

\author{
Chaptrte PREMier
}

(suite)

Les jeunes gens qui sont assez âgés pour contracter mariage validement, plus de quatorze ans pour les garçons et plus de douze ans pour les filles, doivent aussi, sous le Régime Français, obtenir le consentement de leurs parents. Cette obligation remonte à 1556, alors que le roi Henri II statuait:

que les enfants qui contracteraient de mariages clandestins contre le gré de leur père et mère pourraient être exhérédés (déshérités). Il ajoutait cependant que les fils excédant l'âge de trente ans et les filles ayant vingt-cinq ans passés, du moment qu'ils se seraient mis en devoir de requérir l'avis et conseil de leur père et mère ne seraient pas tenus d'attendre leur consentement. ${ }^{60}$

L'ancien droit français prévoyait donc le cas où les parents pourraient s'opposer au mariage de leurs enfants âgés de plus de vingt-cinq ans; ces derniers n'étaient pas tenus d'attendre le consentement pourvu qu'ils aient requis « l'avis et conseil de leur père et mère », ce qui se faisait par les sommations respectueuses. L'article 123 de notre Code Civil se lit comme suit: «Les sommations respectueuses aux père et mère ne sont plus obligatoires. ${ }^{61}$ Cependant, sous le Régime Français, l'obligation existait encore, et les jeunes gens, placés dans les conditions décrites plus haut, devaient faire rédiger par un notaire trois sommations respectueuses, requérant poliment le consentement des parents; la plupart du temps, comme nous le constatons, il s'agissait de suppliques plutôt que de sommations. Les greffes des notaires en contiennent quelques exemples; nous citons les trois

* Voir notre Revue, XIII : 230-246.

60 Pierre-Georges Roy, Les Petites Choses de notre Histoire, I: 172.

๑1 René Deguire, Code Civil de la Province de Québec, 35. 
sommations respectueuses de Jean-Claude Louet à son père, le notaire Claude Louet, en janvier 1733; le jeune Louet avait alors trente ans et il voulait épouser la fille d'un cordonnier; son père, personnage important, s'opposait à cette union; voici les suppliques :

Mon très cher père,

Je suis dans la dernière des désolations de me voir privé des douceurs que j'avais coutume de gouter auprès de vous; j'éprouve avec une douleur extrême aujourdhuy que vos mouvements de tendresse dont j'ay esté tant de fois sensiblement touché sont entièrement étins (sic); cependant, mon cher père, si je m'abstrait de l'obéissance et de la soumission qui vous est due c'est l'obligation indispensable de réparer la réputation de celle que j'ay entierrement perdu, sans quoy il n'y a point de salut pour moy.

Enfin, mon cher père, je vous conjure par votre amour paternel et par tout ce que vous avez de plus cher pour votre propre sang de vouloir vous laisser fléchir et touché du sort fâcheux de la pauvre fille et de la situation pitoyable où je suis réduit depuis si long temps. Vous avez parlé, j'ay obéi, vous m'avez éloigné d'auprès de vous, envoyé dans un endroit où je n'avais pour consolation que des pleurs et des soupirs qui faisaient une continuelle compagnie.

Je crois enfin que c'est aujourd'huy que touché de mes meaux (sic) vous voudrez bien m'accorder la grâce que je vous demande.

Celuy qui est,

Mon cher père,

Votre très humble et très soumis fils,

C. Louet. ${ }^{62}$

Cette sommation est une supplique d'un fils respectueux, qui explique sa décision, tout en essayant de convaincre son père. Il a fait des promesses et maintenant il est trop tard pour reculer: c'est une question de salut! C'est ce qu'il affirme dans la seconde sommation, tout aussi pathétique que la première:

62 Les sommations respectueuses, dans RAPQ, (1921-1922) : 60 . 
Rien ne peut arrester le cours de mon affliction de voir votre cœur toujours inflexible et irrévocable à toutes mes supplications et soumissions. Serait-il possible que la tendresse autrefois sy grande pour votre enfant se serait changé en une disgrâce perpétuelle ? Je ne vois point cependant en telle rencontre que j'aie pu mériter votre haine. Tous vos sentiments sont toujours fortement opposés aux miens, ce quy me désolle entièrement. Cependant, mon cher père, je suis dans une obligation indispensable d'accomplir les promesses que j'ay faittes sy je veux assurer mon salut. Je vous supplie donc très humblement, mon cher père, de me regarder encore comme votre propre enfant et de m'accorder la grâce que je vous demande avec toute la soumission possible, mon très cher père.

Votre très humble et très soumis fils,

Louet. ${ }^{63}$

Cette deuxième sommation est accompagnée d'une attestation de l'huissier du Conseil Supérieur:

L'an mil sept cent trente trois, le vingt-troisième jour de janvier avant-midy, à la requeste du d. Claude Louet fils quy fait élection de domicile en cette ville en la maison du s. Larchevesque, boucher, sise rue St-Jean, J'ay huissier au Conseil Supérieur de Québec, soussigné y résidant rue du Sault au Matelot signiffié la lettre dont coppie est cy-devant transcrite à Me. Claude Louet père, notr. Royal en la prevosté de cette ville, y demeurant en parlant à sa personne à domicille à ce qu'il n'en ignore. Fait et laissé ces présentes les jour et an susdits.

De Saline. ${ }^{64}$

Le notaire Louet semble ne pas avoir été tellement touché par les suppliques de son fils; en effet, le lendemain son fils lui faisait parvenir une dernière sommation:

Mon cher et honoré père,

Pénétré de la plus vive douleur j'ay recours à cette tendresse paternelle dont j'ay senty tant de fois les doux mouvements. Serait-il possible, mon

63 Ibid., 61.

64 Loc. cit. 
cher père, que vous m'en priveriez dans un temps (où) elle me serait sy essentielle? Non, je suis certain que vous êtes trop amateur du salut des âmes pour me désaprouver en ce que je persiste.

J'aurais eu cependant lieu d'espérer que vous vous fussiez fléchi par les deux précédentes soumissions que jay eu l'honneur de vous faire sans comprendre la lettre que j'ay pris la liberté de prier Mr. Plante de vous la représenter.

C'est donc par celle-cy, mon très cher père, que je vous conjure et voue prie très humbelment (sic) de vous laisser fléchir et m'accorder votre consentement au mariage de celuy quy est avec toutte la soumission et le respect possible votre très humble et soumis fils

Louet l'ainé. ${ }^{65}$

Cette dernière sommation est accompagnée, elle aussi, d'une attestation de l'huissier De Saline, semblable à la précédente. Comme nous le constatons par ces textes, les sommations respectueuses étaient une procédure un peu curieuse: d'un côté nous avons un père qui ne veut pas se laisser fléchir, et de l'autre, un fils qui, malgré le style affecté du tabellion, est bien décidé à passer outre au désir paternel. Les textes des autres sommations respectueuses conservées dans les greffes sont généralement plus élaborés; c'est pourquoi nous avons choisi ceux-ci, qui nous donnent une bonne idée de la procédure, que les majeurs doivent suivre avant de se marier, si leurs parents refusent leur consentement. De plus les sommations faites sont notées dans le registre; ainsi à Beauport, le 10 avril 1752, il est dit:

$\mathrm{Vu}$ les trois sommations faites à la d. Angélique Landry, mère de la $d$. future espouse (.....) pour obtenir par la d. Vachon la permission de se marier, auxquelles sommations il n'auroit rien été répondu sinon que elle ne vouloit pas donner son consentement, mais que sa fille étoit en age et qu'elle pouvoit faire ce qui luy plairoit. ${ }^{66}$

Pour se marier, les majeurs doivent obtenir le consentement de leurs parents ou bien suivre la procédure légale des trois som-

${ }^{65}$ Loc. cit.

${ }^{66}$ Langevin, op. cit., 186. 
mations respectueuses. Quand il s'agit de mineurs, il leur faut le consentement de leurs parents ou de leurs tuteurs:

Les mineurs qui voudront contracter mariage, soit en France soit dans les colonies françaises, ne pourront le faire sans l'avis et le consentement par écrit du tuteur nommé dans le pays où le père avait son domicile au jour de son décès, sans néanmoins qu'il puisse donner le dit consentement que sur l'avis des parents qui seront assemblés à cet effet par devant le juge, qui l'aura nommé tuteur. ${ }^{67}$

Le consentement des parents doit donc être donné par écrit et, si les parents sont morts, le tuteur ne peut donner son consentement sans avoir consulté le conseil de famille. Dès 1627, un certain sieur Berthelot, mineur venant de Paris, voulait épouser une demoiselle Roussel, sans avoir par écrit le consentement de son père:

Nous lui avons déclaré que son témoignage ne suffisait pas, et que les ordonnances défendaient de prouver par témoins et autrement que par écrit de leur main ou devant notaire les consentements des pères et mères en tel cas requis. ${ }^{68}$

Plus tard, en 1741, le Conseil Supérieur rend nuls les mariages des mineurs faits sans le consentement de leurs parents; René-Ovide Hertel de Rouville, mineur, a épousé Louise André de Leigne, et, à la demande de la mère du garçon, le Conseil Supérieur:

déclare le dit mariage non valablement contracté; Fait defenses au dit sieur de Rouville et à la dite Demoiselle André de prendre la qualité de mari et de femme, et de se hanter et fréquenter, sous les peines du droit;

fait défenses à tous notaires de passer des contrats de mariage de mineurs que les dits mineurs ne soient duement assistés et autorisés de leurs pères, mères, tuteurs ou curateurs, qui signeront au dit contrat (...)

enjoint pareillement à tous curés et prêtres, tant séculiers que réguliers, de marquer dans les actes

${ }^{67}$ Edits et Ordonnances, I: 440.

68 Ibid., II : 312. 
de célébration de mariage si les contractants sont enfans de famille, en tutelle ou curatelle ou en la puissance d'autrui, d'y énoncer pareillement les consentements de leurs dits pères et mères, tuteurs ou curateurs. ${ }^{69}$

Même si le roi, dans son désir de peupler la colonie, encourage les jeunes gens à se marier très jeunes, il leur faut obtenir le consentement de leurs parents, sinon le Conseil Supérieur peut annuler leur mariage. Enfin si le futur époux est militaire, il lui faut une permission spéciale du gouverneur et de son capitaine; ces permissions sont notées dans les registres; c'est ainsi que nous lisons dans le registre de la paroisse de Beauport, en date du 7 janvier 1713:

Mariage entre Pierre Mons fils d'Etienne, et de Marie Pie (...) natif de la paroisse de St. Martin, du bas Limousin, demeurant dans ce pays depuis dix neuf ans dans la ferme de la Mareché, étant à présent Caporal dans la compagnie de Monsieur Begon ayant obtenu et montré à nous la permission de Monsieur de Vaudreuille general; et de Mr. Begon son Capitaine. ${ }^{70}$

Le 29 janvier 1725 :

Jean Massé aiant obtenu de Monsieur le Marquis de Vaudreuil Gouverneur et Lieutenant-Général pour le Roy en la Nouvelle-France la permission de se marier. ${ }^{71}$

Le 23 septembre 1748:

Nicolas Philbert Bouchard dit Jolycoeur soldat de la compagnie de Raimond détaché dans les canoniers fils de feu Antoine Bouchard et Marie Anne Donzers de la paroisse de St. Severin ville et archevesché de Paris (...), et Catherine Vallée (...) vue (...) la permission (...) donnée par Monsieur le Marquis de la Gallissonnière. ${ }^{72}$

Le 26 avril 1751 :

Mariage entre François Tiriot, soldat de la Compagnie de St. Ours (...) et Elizabeth Tardif

69) Ibid., II : 208s.

70 Langevin, op. cit., 103.

71 Ibid., 146.

72 Ibid., 180. 
(....) permission visée par Mr. de Ramezay, major de Québec. ${ }^{73}$

Le 31 janvier 1757 :

Mariage entre J. B. Blavier (....) soldat de la compagnie de Dubouquet au Régiment de Guyenne de présent à Québec (....) et Marie Thereze Brusseau (....) Permission signée Dubouquet, Fonbonne, Montcalm, Vaudreuil et Laparesse, Capne. ayde major. ${ }^{74}$

Ces quelques exemples, parmi un grand nombre, nous montrent bien que les militaires ne pouvaient se marier sans obtenir la permission de leurs supérieurs militaires.

En plus de ces permissions et du consentement des parents, les contractants devaient aussi connaître leurs obligations selon la demande de Monseigneur de Saint-Vallier:

Si les Curez croyent avoir un sujet raisonnable de douter de la capacité de ceux qui demandent à se marier, ils doivent avant de leur conferer ce Sacrement, les interroger sur les principaux Mysteres de la Religion, \& ne les pas marier qu'ils ne soient suffisamment instruits. ${ }^{75}$

Dans un synode, préparatoire au Rituel, tenu en 1698, l'évêque de Québec décrétait ce qui suit:

Afin de remédier à l'inconvénient de l'ignorance qui se trouve en plusieurs de ceux qui cherchent à se marier, et qui ne sont pas instruits des choses nécessaires au salut, Nous défendons à tous les Curés et Missionnaires, de publier le second Ban avant que les Parties intéressées ne leur aient parlé chacune en particulier. ${ }^{76}$

Nous avons dans ces prescriptions de Monseigneur de SaintVallier un programme d'examens prénuptiaux. De la part des contractants sont requis une certaine connaissance religieuse et des dispositions vraiment chrétiennes. Dès 1691, Monseigneur de Saint-Vallier réprimait certains abus:

73 Ibid., 185.

74 Ibid., 189.

75 Mgr de Saint-Vallier, op. cit., 332.

76 Mandements des Evếques de Québec, I: $376 \mathrm{~s}$. 
Comme nous avons été informé que des personnes se présentaient au Sacrement de Mariage sans y apporter les dispositions de piété, de modestie et autres conditions requises, Nous enjoignons à toutes les personnes qui ont à se marier de s'y préparer par l'instruction des choses qui leur sont nécessaires de savoir pour bien recevoir ce Sacrement et surtout de s'en approcher avec piété et dévotion, bannissant toutes les causeries et autres irrévérences qui se commettent quelquefois pour lors dans l'Eglise, comme l'expérience l'a fait remarquer; ce qui est une profanation honteuse d'une chose si sainte, capable d'attirer la malédiction de Dieu sur les personnes mariées. $^{77}$

Il appartient aux pasteurs d'enseigner aux fidèles leurs droits et leurs obligations; Monseigneur de Saint-Vallier leur en fait un devoir:

Il est bien important que les Pasteurs expliquent souvent à leur peuple les dispositions que doivent avoir ceux qui veulent le recevoir; afin qu'un si grand sacrement ne soit pas reçu en péché; Sacrement dont la grace est plus rare, l'usage souvent plus corrompu, l'abus plus contagieux, \& les suites plus scandaleuses. ${ }^{78}$

$\mathrm{Du}$ côté des contractants, plusieurs dispositions sont requises avant de s'approcher d'un si grand sacrement. Monseigneur de Saint-Vallier énumère six dispositions principales que les chrétiens doivent apporter dans la préparation de leur mariage:

$\mathrm{La} 1^{\mathrm{e}}$ disposition est de bien consulter Dieu pour sçavoir s'il les appelle à l'état de Mariage. Ceux qui veulent se marier sans le consulter, dans la vûë de satisfaire plutôt leurs passions dereglées que de lui plaire, s'exposent à agir contre sa volonté, qui les destinoit peut-être à un étât plus parfait, \& à être par leur conduite dereglée malheureux en ce monde \& en l'autre. ${ }^{79}$

Dans une décision aussi grave, les parents ont aussi un rôle important à jouer: c'est un rôle de surveillance, car «il est fort

77 Ibid., I : 276.

78 Mgr de Saint-Vallier, op. cit., 345.

79 Loc. cit. 
à craindre, dit le Rituel, qu'on ne contracte les vices et les injustices des familles ausquelles on s'allie »; plus loin, Monseigneur de Saint-Vallier note bien la lourde responsabilité des parents quand il dit «qu'ils se rendent coupables des incontinences ou autres desordres de leurs enfans, quand par un principe d'avarice, entêtement, ou autres motifs, ils empêchent leurs filles de se marier avant vingt-cinq ans, ou leurs garçons avant trente ans ».

La $2 \mathrm{e}$ chose qu'on doit observer avant de se marier, est de se faire instruire par son Pasteur ou son Confesseur, ou par des personnes de piété, des maximes générales de la Religion, des devoirs particuliers des personnes mariées, (.....)

La $3^{\mathrm{e}}$ est de faire une bonne Confession generale et une sainte Communion en la Paroisse. Il faudroit que les personnes qui veulent se marier, passassent les jours qui précederont leur Mariage dans l'éloignement de tout péché, \& dans la pratique des bonnes oeuvres, prieres, saintes lectures, jeûnes \& aumônes, en l'assistance aux Offices Divins, \& à la Sainte Messe. Ils feront faire des prieres, \& offrir le S. Sacrifice pour eux; afin que Dieu répande ses benedictions sur leurs personnes \& sur leur Mariage. ${ }^{80}$

En plus de ces dispositions pour connaître la volonté de Dieu, s'instruire de leurs devoirs et se préparer saintement à leur mariage, les contractants doivent aussi purifier leur esprit et se présenter à l'autel dans une tenue modeste:

La $4^{\mathrm{e}}$. (disposition c'est) D'avoir une veritable pureté d'intention, ne se proposant dans le Mariage que la gloire de Dieu \& sa propre sanctification, \& non la satisfaction de ses cupiditez, de son ambition, de son avarice $\&$ de ses passions honteuses.

La $5^{\text {e}}$. De tenir son esprit saintement occupé de la grandeur de ce Sacrement le jour de son Mariage, desirant d'en remplir les significations mysterieuses; $\&$ pour ce sujet l'on doit éloigner de soy le plus que l'on peut, toutes les pensées \& les entretiens qui pourroient mettre obstacle aux graces que l'on peut recevoir en ce Sacrement.

La $6^{\text {e }}$. De faire paroitre beaucoup de modestie dans ses habits \& son maintien, se persuadant que

80 Ibid., $346 \mathrm{~s}$. 
le Seigneur ne sanctifiera pas des nôces où l'on veut employer les oeuvres du Démon, qui sont le luxe, la vanité, la gourmandise, la crapule (ivresse, débauche), \& les autres dissolutions où l'on ne se laisse aller que trop ordinairement dans ces jours-là. ${ }^{81}$

Telles sont les principales dispositions que Monseigneur de Saint-Vallier exige des futurs mariés; elles sont empreintes de la sagesse d'un pasteur clairvoyant, qui connaît bien les excès auxquels les jeunes sont exposés. Cette préparation au mariage est un gage de sainteté et de bonheur pour les époux, car Dieu bénit les chrétiens qui prennent de tels moyens pour se sanctifier. Tout est défini avec netteté, tant du côté des pasteurs et des parents que du côté des contractants.

Un autre point auquel les jeunes gens doivent apporter un grand soin, c'est le choix judicieux de leur conjoint; il leur faut sans doute consulter leurs parents mais avant tout demander à Dieu :

la grace de leur faire connoître, si c'est avec la personne que l'on leur propose, plutôt qu'avec une autre, qu'ils doivent se marier; parce qu'il n'y a que Dieu qui puisse apprendre à faire un bon choix. Les parens peuvent donner des richesses à leurs enfans; mais non pas une bonne femme, qu'on doit regarder comme un don des plus prétieux de Dieu. On doit la lui demander comme fit le serviteur d'Abraham qui fut envoyé en Mésopotamie par son maître pour chercher une femme à Isaac, Genes. 24 . Les parens doivent prendre garde que leurs enfans ne s'allient avec des personnes remplies de vices, pleines des désirs du siècle, des vanitez \& des maximes corrompuës du monde, dont les biens soient mal acquis. $^{82}$

Puisque le mariage est un engagement qui dure toute la vie, il faut donc que les jeunes gens qui désirent se marier recherchent un conjoint dont les qualités complètent les leurs ; mais il y a certaines qualités de base sans lesquelles il serait osé de s'aventurer dans le mariage. C'est pourquoi les futurs époux doivent:

81 Loc. cit.

82 Loc. cit. 
prier Dieu de leur faire la grace de trouver la personne avec laquelle ils pourront faire plus aisément leur salut; examiner ensuite si celle qu'on leur propose a les qualitez suivantes; si elle a la crainte de Dieu, si elle est sage, propre à conduire un ménage; sur-tout si si (sic) elle est capable de bien élever des enfans, \& de les instruire des veritez Chrétiennes. ${ }^{83}$

Cette dernière qualité revêt une importance particulière puisqu'un des buts du mariage est d'avoir des enfants et de les élever chrétiennement; la femme doit être capable de conduire un ménage et être sage, car c'est le principal gage de fidélité. De plus, les parents sont les conseillers choisis par Dieu lui-même pour faire connaître sa volonté; les enfants doivent donc:

prendre garde de les contrister en se mariant contre leur volonté. Cependant il faut convenir de bonne foy, que cette marque seule ne suffit pas pour déterminer des enfans à un engagement de toute la vie, ausquel les Peres \& les Meres font quelquefois plus d'instance qu'ils ne devroient sur cela. C'est une verité assurée, qu'ils ne doivent pas les croire, lorsqu'ils ne regardent en ce choix que l'établissement d'une fortune temporelle, sans considerer ce qui leur est utile pour leur salut, voulans souvent les engager à se marier avec des personnes dont la compagnie pourroit nuire à leur sanctification. ${ }^{84}$

Sous prétexte de fréquentations, les rencontres entre jeunes gens peuvent parfois tourner au libertinage; c'est pourquoi, Monseigneur de Saint-Vallier, en 1693, fait parvenir aux curés une ordonnance touchant le sacrement de mariage:

Ayant remarqué que plusieurs jeunes gens, et particulièrement les gens de guerre, sous prétexte de rechercher des filles en mariage, se comportent d'une manière fort licencieuse avec les dites filles, qui se laissent souvent abuser, sous l'espérance de les épouser, dans la persuasion qu'elles ont que les fautes et les accidents qui leur peuvent arriver en ce sujet, seront autant de motifs à leurs parents de poursuivre leurs dits mariages aussi bien qu'aux

83 Ibid., 330.

84 Loc. cit. 


\begin{abstract}
personnes de qui dépendent les gens de guerre, de leur en accorder sans peine la permission; ce qui ne contribue pas peu à entretenir le libertinage et le désordre parmi la jeunesse, au grand scandale du public; Nous pour arrêter le cours d'un si grand mal, défendons très expressément aux curés et autres faisant les fonctions curiales dans notre Diocèse de marier les personnes ci-dessus, à moins qu'ils n'en aient reçu une permission signée de Nous, laquelle Nous déclarons que Nous n'accorderons qu'à ceux que Nous saurons n'avoir causé aucun désordre ni scandale, avec les filles qu'ils voudraient épouser et s'être comportés chrétiennement dans la recherche de leurs mariages..$^{85}$
\end{abstract}

Après le choix du conjoint, sous le Régime Français, avait lieu parfois la cérémonie des fiançailles solennelles; ces fiançailles officielles apportèrent certains abus qui conduisirent Monseigneur de Saint-Vallier à les défendre:

Il a été réglé que tous les curés dans le diocèse, ne feront plus les fiançailles, qui se font avant le Mariage. ${ }^{86}$

Cette défense fut portée le 28 février 1698 par l'évêque de Québec. Quelques années plus tard, en 1703, dans le Rituel, Monseigneur de Saint-Vallier renouvelle cette interdiction tout en y apportant un adoucissement:

Les fautes que Nous avons reconnuës y être commises, Nous ont fait prendre la résolution de les retrancher, jusqu'à ce que Nous ayons pû trouver les moyens d'en exclure les abus. Ainsi ce que Nous venons de dire de cette Solemnité ne sera que pour l'instruction des Pasteurs; afin qu'ils puissent les mettre en usage, lorsqu'il n'y aura aucun sujet de craindre cet abus, \& qu'elles pourront être celebrées avec modestie. ${ }^{87}$

Donc jusqu'à 1698, et même après cette date, en certains cas, les pasteurs pouvaient célébrer des fiançailles solennelles. Il est important de savoir en quoi consistait cette cérémonie et les

85 Mandements des Evêques de Québec, I: $300 \mathrm{~s}$.

86 Ibid., I: 376.

87 Mgr de Saint-Vallier, op. cit., 339. 
obligations qu'elle entraînait. Dans le Rituel, les fiançailles sont définies comme suit:

Les Fiançailles ne sont autre chose qu'une promesse solemnelle que deux personnes de different sexe se font l'une à l'autre de se prendre pour Mary $\&$ pour Femme. ${ }^{88}$

Cette promesse solennelle ne peut se faire qu'entre des personnes capables de contracter validement un mariage; de plus cette cérémonie revêt une solennité comparable à celle du mariage lui-même, car les fiançailles doivent se faire à l'église, en présence du curé et des témoins:

La promesse qu'on fait aux Fiançailles étant l'expression d'un consentement libre, il est necessaire que les parties s'expliquent nettement, \& en des termes qui ne permettent pas de douter de la sincerité de leurs promesses.

Les Fiançailles doivent être faites en l'Eglise, en presence du Curé \& des Témoins. Comme elles imposent une obligation qu'on doit accomplir, \& qu'on ne peut violer sans commettre quelque peché, elles ne peuvent être faites que par des personnes, qui ont atteint l'âge de la raison, \& qui connoissent à quoy elles s'engagent. ${ }^{89}$

Cette promesse solennelle ressemble beaucoup à l'échange de consentement matrimonial, mais elle n'est que la promesse mutuelle de donner ce consentement. Ce n'est pas un mariage, c'est pourquoi les fiancés sont obligés de prendre certaines mesures de prudence:

Les Curez auront soin de défendre aux Fiancez de demeurer dans une même maison, ni de se fréquenter familierement. Le different usage que Nous avons remarqué être dans plusieurs Paroisses de ce Diocese, dans le plus grand nombre desquelles on n'observoit point la solemnité des Fiançailles, \& dans les autres, on l'observoit. ${ }^{90}$

Cette dernière remarque de Monseigneur de Saint-Vallier nous montre que le nombre de ces fiançailles n'était pas tellement

88 Loc. cit.

89 Loc. cit.

${ }^{90}$ Loc. cit. 
élevé, puisque le plus grand nombre des paroisses n'observaient point la solennité des fiançailles; cet échange de promesses entraîne un empêchement prohibant, c'est-à-dire rendant le mariage illicite entre le fiancé et les parentes de sa fiancée, de même qu'entre la fiancée et les parents de son fiancé; le Rituel note cet empêchement ainsi :

Les fiançailles contractées avec une autre personne, qui n'ont point été résoluës par autorité légitime, ou par un commun consentement. ${ }^{91}$

Ces fiançailles proprement dites ne peuvent donc être annulées que par un acte de l'autorité légitime ou encore par le mutuel consentement annulant la promesse mutuelle. De plus les fiançailles solennelles peuvent constituer un empêchement dirimant, c'est-à-dire rendant le mariage nul; c'est un empêchement d'honnêteté:

Honestas, est un empêchement qui vient de la promesse publique; par exemple, que Pierre \& Marie se sont faites de s'épouser; ce qui empêche que Pierre, si Marie vient à mourir, ou à se faire Religieuse, ne puisse épouser la Mère. la Sœur \& la Fille de Marie, à cause de l'honnêteté publique. ( .....) L'empêchement qui vient d'une promesse publique, est restraint au premier degré..$^{92}$

Dans les registres de la paroisse de Beauport, le 15 octobre 1685, nous trouvons un exemple de fiançailles solennelles:

Mariage après dispense de 2 bans et les fiançailles faites en face d'Eglise, de Jean, fils de Mre. Edmond Gaultier et de Damoiselle Magdeleine Jahan, de la parroisse de St. Eloy Ville et Archevesché de Bordeaux d'une part et Marie fille de François Guyon desprez et de Marie Magdeleine Marsolet, de cette parroisse, d'autre part. ${ }^{93}$

Comme nous pouvons le constater, les fiançailles solennelles, sans être fréquentes, existaient sous le Régime Français, surtout avant 1698. Elles étaient une manière solennelle d'annoncer un mariage. En plus des fiançailles, quand elles existaient, il y

91 Ibid., 338.

92 Ibid., 335.

83 Langevin, op. cit., 40. 
avait une autre annonce des mariages qui était obligatoire, c'étaient les publications. Voici comment le Rituel les définit:

La Publication des Bans est une Publication que les Curez font au peuple, qu'il y a promesse de Mariage entre telles \& telles personnes, pour découvrir si elles ne sont point engagées dans quelqu'un des empêchemens qui pourroient faire obstacle à leurs Mariages. ${ }^{94}$

Le but des publications est donc bien précis, c'est de découvrir les empêchements de mariage. A cause de leur importance, les publications ne peuvent se faire n'importe quand ou n'importe comment. Monseigneur de Saint-Vallier détermine les circonstances de lieu et de temps qui entourent les publications:

Les Curez ne doivent publier les Bans qu'à la priere des Parties, \& du consentement des Peres \& Meres, Tuteurs ou Curateurs, si ce sont des enfans de Famille.

Les Bans ne peuvent être publiez que dans l'Eglise Paroissiale. Ces Publications doivent se faire de telle maniere qu'il y ait au moins deux ou trois jours francs entre chacune, par trois Dimanches ou Fêtes chômées, au Prône de la grande Messe, \& non pas aux Vêpres, ni aux Messes de Confrairies.

L'on n'en doit délivrer le Certificat que 24. heures aprés (sic) la derniere Publication. ${ }^{95}$

Il faut donc que les parents donnent leur consentement avant d'annoncer les publications; comme nous l'avons vu précédemment, le mariage ne peut ordinairement se faire sans ce consentement; c'est pourquoi il serait inutile de publier un mariage alors que les parents ne consentent pas. Certaines difficultés peuvent se présenter au sujet de la paroisse dans laquelle les publications sont obligatoires; Monseigneur de Saint-Vallier donne les instructions suivantes:

Que lorsque les deux Parties sont de Paroisses differentes, la Publication des Bans se fasse dans les deux Eglises, au lieu où chacune des Parties habite actuellement; de maniere que le Curé qui doit

94 Mgr de Saint-Vallier, op. cit., 339.

95 Ibid., 340. 
celebrer le Mariage ne donne point la Benediction Nuptiale, qu'il n'ait vû un Certificat de la Publication des Bans faite dans l'autre Paroisse.

Que lorsque les Parties n'ont pas demeuré dans la Paroisse où elles veulent se marier, au moins l'espace de six mois, elles sont obligées de faire publier leurs Bans dans celle d'où elles sont sorties, \& de rapporter un Certificat signé du Curé. La même regle doit être observée à l'égard des personnes qui ont deux domiciles dans deux Paroisses differentes, leurs Bans doivent être publiez dans l'une \& dans l'autre Paroisse. ${ }^{96}$

Les publications sont obligatoires dans tous les cas, à moins d'avoir obtenu une dispense de l'évêque; si les publications se font ailleurs que dans l'église où le mariage a lieu, le curé doit exiger un certificat ainsi rédigé:

Je N. Curé de N. soussigné, certifie à tous ceux qu'il appartiendra, que j'ay publié au Prône de la Grande Messe, à trois Dimanches (ou Festes non consécutives) les Bans entre N.N. fils de N.N. \& de N.N. ses pere \& mere, mes Paroissiens, d'une part. Et N.N. fille de N.N. \& de N.N. ses pere \& mere, de la Paroisse de N. d'autre part. J'atteste de plus que ledit N. mon Paroissien, s'est confessé \& qu'il a communié le jour du mois de : qu'il est suffisamment instruit de la Doctrine Chrétienne \& du Sacrement de Mariage: \& que je n'ay découvert aucun empêchement à leur futur Mariage. En foy de quoy j'ay délivré le présent Acte. ${ }^{97}$

L'évêque peut dispenser d'une publication, et même des trois, toutes les fois qu'il le juge à propos; cependant il ne faut pas abuser et les raisons doivent être suffisantes:

Lorsque les Parties ont pris dessein d'obtenir Dispense de quelques Bans, ou lorsqu'elles l'ont obtenu en effet, l'on en fera mention dans la Publication qu'on fera.

Nous sommes touchez de l'erreur où sont les Peuples, lors qu'ils croyent que les Evêques peuvent aussi facilement dispenser leurs Diocesains de faire

96 Loc. cit.

97 Ibid., 645s. 
publier les Bans de leur Mariage, qu'ils le demandent souvent sans aucune raison légitime; Nous déclarons que Nous n'aurons point d'égard ni à la vanité de ceux qui ne demandent ces Dispenses que pour se distinguer du commun, ni à la délicatesse des autres, qui ne sçauroient souffrir qu'on publie leurs noms dans les Eglises, ni à la proximité du temps de l'Avent \& du Carême, à moins que Nous ne connoissions évidemment, qu'on n'a pas affecté d'attendre exprés (sic) à cette extrémité, \& que le retardement pourroit causer un dommage notable aux Parties interessées. ${ }^{98}$

Avant le Rituel, dans un synode tenu à Ville-Marie le 11 mars 1694, une recommandation semblable avait été faite:

Nous invitons les Curés et Missionnaires à faire connaître à leurs Paroissiens qu'ils ne doivent pas demander si facilement la dispense de la publication des Bans de mariage, étant une règle établie par le Saint Concile de Trente, dont on ne doit pas se dispenser sans grande raison..$^{99}$

De plus il y a des droits à payer pour la publication des bans de mariage; ils ont été fixés au premier synode de Québec, le 9 novembre 1690 :

Croyant aussi nécessaire de fixer les droits qu'on peut prendre pour la publication des Bans de Mariage, et la Messe qu'on doit dire, nous avons réglé qu'on pourra prendre six livres pour les deux ensemble: et que pour la publication seule des Bans, on ne prendra que quarante sols. ${ }^{100}$

En 1703, Monseigneur de Saint-Vallier a demandé à la Sacrée Congrégation de la Propagation de la Foi:

Quaeritur utrum Barbaris danda sit dispensatio generalis a Proclamationibus? - Doit-on donner aux Sauvages une dispense générale des Publications?

- Resolutio: negative; réponse: non. ${ }^{1}$

Le Concile de Trente a demandé de faire des publications pour éviter les mariages nuls en raison des empêchements peu

98 Ibid., 340s.

99 Mandements des Evêques de Québec, 318s.

100 Ibid., 273.

1 Ibid., 461. 
connus; c'est pourquoi, Mgr de Saint-Vallier restreint les dispenses dans les limites raisonnables, en ne se laissant pas émouvoir par la sensiblerie ou l'orgueil de certains diocésains. Dans les registres de Beauport, nous avons relevé plusieurs cas de dispenses de deux bans: en 1689, 1690, 1692, 1717. ${ }^{2}$ De plus nous avons rencontré deux cas de dispenses des trois bans; le 26 décembre 1708:

Dispense de tous bans et de tems, par Monsr. De La Colombiere Grand Vicaire (...) Mariage entre Guillaume La Vigne fils de Pierre (...) et de Marie La Roche de la parroisse de Brassard Dioceze de Cahors Soldat (...) et Marie Jeanne Parenteau. ${ }^{3}$

L'autre cas est en date du premier octobre 1727, dans l'après-midi :

Mariage entre Basile Bonnau ( . . . ) de St. Francois dans l'ile St. Laurens (...) et Marie Magdeleine Parent $(\ldots)$ après avoir dispensé de la publication des trois bancs (sic) (Signé) Jean eveque de quebec. $^{4}$

En parcourant les registres de la paroisse de Sainte-Foy, nous avons remarqué un cas de dispense des trois bans; il s'agit du mariage de Denis Mallet et de Geneviève Léonard, le 10 novembre 1699.5 Par ces quelques exemples, nous pouvons constater que les dispenses des publications ont été plutôt rares sous le Régime Français; Monseigneur de Saint-Vallier s'est montré sévère sur ce point et c'est à juste titre.

Les publications se font ordinairement en vue d'un mariage prochain; c'est pourquoi le Rituel annule leur effet:

Si aprés la publication des Bans les Parties laissent passer trois mois sans se marier, elles seront obligées de faire publier de nouveau trois autres Bans avant de se marier, à moins que Nous ne jugions à propos de les en dispenser. ${ }^{e}$

2 Langevin, op. cit., 45, 46, 50, 110.

3 Ibid., 84.

4 Ibid., 149.

5 Registre de la Paroisse Notre-Dame de Foy, 1699.

6 Mgr de Saint-Vallier, op. cit., 341. 
Une formule spéciale est donnée par Monseigneur de SaintVallier pour que les publications soient faites partout dans les mêmes termes. Après l'énumération des noms des contractants et de leurs père et mère le curé doit ajouter:

Nous vous avertissons tous en general \& en particulier, que si quequ'un de vous sçait qu'il y ait quelque empêchement Canonique entre ces personnes, qui ne leur permette pas de contracter Mariage ensemble, il est obligé de Nous en avertir sous peine d'Excommunication: Ce que Nous vous faisons sçavoir pour la premiere, seconde, ou troisiéme fois. Cependant Nous vous exhortons de prier Dieu qu'il répande ses graces \& ses benedictions sur ces personnes qui doivent contracter Mariage ensemble. ${ }^{7}$

Si les personnes qui veulent se marier ont déjà obtenu une dispense, le curé doit le dire à la suite de la publication. ${ }^{8}$ L'obligation d'avertir que l'on connaît un empêchement est très grave puisque la peine encourue est l'excommunication. Cette peine est définie dans le Rituel:

Une Censure Ecclesiastique, qui retranche certains Fideles du Corps de lEglise (...) \& qui les prive du droit qu'ils avoient aux biens communs de tout le corps de l'Eglise, avant qu'ils en fussent separez. ${ }^{9}$

La dénonciation d'un empêchement canonique doit se faire par écrit selon les prescriptions de Monseigneur de Saint-Vallier :

Si quelqu'un vouloit se rendre opposant à la celebration du Mariage, dont on publie les Bans, le Curé doit lui demander son opposition par écrit, \& la lui faire signer. En cas que l'Opposant ne sçeût pas signer, il doit en faire mention dans l'Acte qu'il dressera, \& le faire signer par deux Témoins, \& ensuite renvoyer les Parties par-devant Nous ou Nôtre Official, pour faire vuider devant lui l'opposition sans delai. Il ne continuëra pas cependant les Publications que l'opposition ne soit levée. Si on ne vouloit pas donner cette opposition par écrit, le Curé passera outre sans y avoir égard.

7 Loc. cit.

8 Ibid., 396.

9 Ibid., 144. 
Le Curé doit avoir égard aux révelations des empêchemens qu'on voudroit faire, encore qu'elles ne soient pas signées \& mises par écrit, \& faire les diligences nécessaires pour en découvrir la verité. S'il trouve la chose douteuse, il Nous consultera auparavant de passer outre. ${ }^{10}$

Ainsi les curés ont le devoir de rechercher avec diligence les empêchements de mariage afin d'éviter les mariages nuls. Un des principaux moyens mis à leur disposition, c'est la publication officielle annonçant qu'il y a promesse de mariage entre un garçon et une fille, que très souvent les paroissiens connaissent parfaitement. Il y a deux sortes d'empêchements: les uns rendent le mariage nul et invalide, ce sont les empêchements dirimants; les autres ne rendent le mariage qu'illicite, ce sont les empêchements que nous appelons prohibants. D'après le Rituel de Monseigneur de Saint-Vallier, les empêchements dirimants sont au nombre de quatorze:

Le premier de ces Empêchemens qu'on appelle erreur, est lorsqu'on est trompé en la personne même que l'on épouse. ${ }^{11}$

L'erreur doit porter réellement sur la personne et non pas sur les circonstances comme la richesse, la virginité, la noblesse: l'erreur de circonstance ne rompt pas le mariage.

L'Empêchement qui vient de la condition est lorsqu'une des parties est trompée sur l'état de l'autre (...) Si Pierre épousant Marie, la croyoit d'une condition libre, \& qu'elle fût Esclave, il n'y auroit point de Mariage; mais il n'y a pas lieu à cet empêchement en ce Royaume, où toutes les personnes sont libres. ${ }^{12}$

Un empêchement dirimant vient du vœu solennel de chasteté ou de religion; le vœu simple ne rompt pas le mariage mais le rend illicite; il y a exception pour le vœu simple fait à la pro-

10 Ibid., 341.

11 Ibid., 333.

12 Loc. cit.

N.B. Nous avons omis intentionnellement de faire le rapprochement avec la législation canonique actuelle sachant que le lecteur pourra le faire lui-même. 
fession religieuse chez les Jésuites ; par ce vœu le mariage est rendu nul.

La parenté ou consanguinité, en ligne directe, rend toujours le Mariage nul, (...) en ligne collaterale, elle le rend nul seulement jusqu'au quatriéme degré inclusivement. Selon cette regle, voici les 4 . Degrez défendus: Frere \& Sœur. Cousin germain $\&$ Cousine germaine. Cousin \& Cousine remuez de germains. Cousin \& Cousine issus de remuez de germain. (...) Il faut observer que lorsque la parenté est de divers degrez, le degré le plus éloigné attire l'autre $(\ldots)$ si Pierre est au troisiéme degré, \& Catherine à laquelle il veut se marier, est au cinquiéme degré, ils peuvent se marier sans dispense. ${ }^{13}$

L'empêchement de crime rend aussi le mariage nul; il s'agit ici de l'homicide et de l'adultère commis selon certaines circonstances:

L'homicide est un empêchement diriment en deux cas. 1. Lorsque Pierre a conspiré avec Marie qu'il veut épouser, de faire mourir Catherine sa femme, \& que la conspiration a eu effet. 2. Lorsque Pierre aprés avoir commis adultere avec Marie, fait mourir Catherine, dans l'intention d'épouser Marie, quoique sans la participation de Marie, en ces deux cas le crime rend le mariage nul.

L'adultere le rend nul aussi en deux cas. 1 . Lorsque Pierre commet adultere avec Marie, avec promesse de l'épouser, si Catherine sa femme vient à mourir, quand la promesse est acceptée par Marie qui sçait que Pierre est marié. 2. Lorsqu'il épouse ladite Marie, laquelle comme lui sçait bien que Catherine sa femme n'est pas morte, Pierre ne peut plus épouser Marie aprés la mort de Catherine. ${ }^{14}$

La différence de culte entre une personne baptisée et une autre non-baptisée rend le mariage nul et Monseigneur de SaintVallier ajoute :

Nous défendons aux Prêtres de nôtre Diocese de marier les Catholiques avec les Heretiques, sous peine de Suspense. ${ }^{15}$

13 Ibid., 333s.

14 Ibid., 334.

15 Loo. cit. 
La liberté est essentielle au mariage de sorte que la violence le rend nul. C'est pourquoi ceux qui sont forcés de se marier, soit directement ou indirectement, pourvu que la contrainte soit injuste, contractent un mariage invalide. De même les ordres sacrés de la prêtrise, du diaconat et du sous-diaconat causent un empêchement dirimant. De plus, en se mariant, une personne contracte un lien, qui l'empêche de se remarier validement, tant que son conjoint n'est pas mort. Le Rituel ajoute cependant:

L'on doit remarquer ici deux choses. La premiere, que quoique le lien du Mariage ne puisse pas être rompu, on peut cependant séparer des personnes mariées, d'habitation, de lit \& de bien: ce qui ne se doit jamais faire cependant que par une Sentence Juridique. La seconde, qu'un mariage qui n'est que ratifié, \& non pas consommé, peut-être dissous par l'entrée en Religion de l'une des deux parties; de maniere qu'aprés sa Profession, la personne qui reste dans le monde est libre de se remarier. ${ }^{16}$

L'empêchement d'honnêteté peut venir d'une promesse publique de mariage: il s'arrête alors au premier degré; il vient aussi d'un mariage ratifié et non consommé: l'empêchement se rend dans ce cas jusqu'au quatrième degré inclusivement. Un autre empêchement assez complexe, c'est l'affinité:

L'alliance ou affinité se contracte par la consommation du Mariage, \& se rencontre entre le Mari $\&$ les Parens de sa Femme; de maniere que cette alliance empêche que le Mary ne puisse épouser les Parens de sa Femme jusqu'au quatriéme degré inclusivement. 17

Il en est de même du côté de la femme par rapport aux parents de son mari. L'affinité qui vient de la cohabitation illicite n'empêche le mariage que jusqu'au second degré inclusivement, par exemple, jusqu'à la cousine germaine inclusivement. Il y a de plus l'affinité spirituelle et l'affinité légale:

L'alliance Spirituelle se contracte par le moyen du Sacrement de Baptême. Elle est entre celui ou celle qui baptise, \& la personne Baptisée; entre celui 
ou celle qui baptise, \& le Pere \& la Mere du Baptisé; entre la (sic) Parain \& la Mere du Baptisé; entre la Maraine \& le Pere du Baptisé; entre les Parain $\&$ Maraine, \& le Baptisé. On ne contracte point cette alliance, lorsqu'on ne baptise pas, \& qu'on supplée seulement les Ceremonies du Baptême. La même alliance se contracte par le moyen du Sacrement de la Confirmation. L'alliance legale est fondée sur l'adoption, \& forme un empêchement selon le Droit Canonique entre le Frere et la Sœur par adoption, tout le temps qu'ils sont en la puissance de celui qui les a adopté. Elle se rencontre aussi entre le Pere qui adopte \& la Fille qui est adoptée, qu'il ne peut pas épouser, même aprés son émancipation, non plus que les Filles qui descendent d'elle en ligne directe. Elle est encore entre celui qui adopte \& la Femme de l'adopté. ${ }^{18}$

L'empêchement d'impuissance vient «lorsqu'une des deux parties ne peut pas consommer l'action du Mariage avec l'autre ». ${ }^{19}$ Il s'agit ici de véritable impuissance et non pas de stérilité ; cette impuissance doit être antécédente au mariage et perpétuelle pour constituer un empêchement dirimant.

Tous les empêchements dont nous avons parlé viennent du droit ancien; le Concile de Trente en a ajouté deux autres: le rapt et la clandestinité. Il y a empêchement de rapt « tant que la personne ravie est en la puissance du ravisseur. Si elle consent volontairement à épouser celui qui l'a ravie, quand elle est mise en liberté, il n'y a plus d'empêchement $\gg^{20}$

La Clandestinité est lorsque le Mariage a été fait en l'absence du Curé \& de deux, ou trois Témoins: il est nul par ce défaut de formalité. ${ }^{21}$

La présence du curé au mariage a été rendue nécessaire par le Concile de Trente. ${ }^{22}$ Les empêchements dirimants sont ordinairement connus, au moins après les publications, mais il peut arriver qu'un empêchement dirimant soit découvert après la

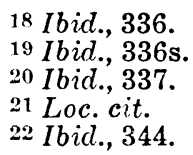


consommation du mariage; il se pose alors des problèmes assez compliqués que Monseigneur de Saint-Vallier résout de la façon suivante:

Si l'empêchement est public, le Curé doit obliger les Parties de se séparer de lit, \& même d'habitation, jusqu'à ce qu'elles en ayent obtenu Dispense, \& fait rehabiliter leur Mariage. Si l'empêchement est secret, \& n'est connu que de l'une des deux parties, il doit lui ordonner de s'abstenir de l'usage du Mariage, \& l'engager à le faire agréer à l'autre, sans lui en dire pour cela la cause, \& ne lui point rendre cependant le devoir du Mariage, ni permettre aucunes libertez, jusqu'à ce que l'empêchement ait été levé par l'Eglise. Si l'empêchement est connu des deux parties, il doit leur déclarer à l'une \& à l'autre, qu'elles ne peuvent plus user du Mariage, jusqu'à ce qu'elles en ayent obtenu Dispense.

Si Nous jugeons à propos de l'accorder, le Curé exigera des parties un nouveau consentement, même en face de l'Eglise, si l'empêchement est public; \& si l'empêchement est secret, il suffit que les parties donnent ce nouveau consentement en particulier \& sans ceremonie en presence de leur Curé.

Si l'empêchement est d'une nature que l'on n'en puisse pas obtenir Dispense, comme s'il vient d'impuissance, il faudra obliger les parties à se séparer l'une de l'autre pour toûjours.

En toute cette matiere il est necessaire que les Curez ne fassent rien sans Nôtre participation, ou celle de nos Grands-Vicaires. ${ }^{23}$

Les empêchements dirimants que nous venons d'étudier peuvent se résumer dans ces vers latins:

Error, conditio, votum, cognatio, crimen, Cultus disparitas, vis, ordo, ligamen, honestas.

Si sis affinis, si forte coïre nequibis ;

Si Parochi, \& duplicis desit præsentia testis. Raptave fit mulier, nec parti reddita tutæ. Hæc facienda vetant Connubia, facta 
Il y a donc quatorze empêchements dirimants, c'est-à-dire qui empêchent de contracter un mariage valide; si on les découvre après le mariage, ce dernier est aussi invalide et nul. Les autres empêchements, appelés prohibants, rendent le mariage, ou son usage, illicite. Ce sont:

Le Vœu simple de Chasteté, ou d'entrer en Religion, ou de ne se point marier. La défense que fait l'Eglise, de celebrer le Mariage depuis le I. Dimanche de l'Avent, jusqu'à la Fête de l'Epiphanie; \& depuis le Mercredy des Cendres, jusqu'au Dimanche de Quasimodo. Les Fiançailles contractées avec une autre personne, qui n'ont point été résoluës par autorité légitime, ou par un commun consentement. (...) Lorsque l'un des Conjoints; par exemple, le Mary a commis un inceste avec la Parente de sa Femme: ou bien vicissim (inversement) la Femme avec le Parent du Mary. Lorsqu'on a baptisé son Enfant soy-même sans necessité, ou que l'on a été son Parain. ${ }^{25}$

Tels sont donc les empêchements qui peuvent s'opposer au mariage: les uns sont dirimants et rendent le mariage absolument nul, les autres sont prohibants et ne le rendent qu'illicite. Si l'on découvre un empêchement, dont on peut obtenir la dispense, il faut alors la demander avant d'aller plus loin et de célébrer le mariage. Généralement c'est l'évêque qui accorde les dispenses; parfois la dispense vient de Rome et en voici un exemple rencontré dans les registres de la paroisse de Beauport:

Le douzieme Octobre (1711) en vertu de dispense accordée par Notre Saint Père le Pape Clément Onzième pour le $3 \mathrm{e}$ degré de parenté (consanguinité), de deux bans par Monsieur Glandelet Grand Vicaire de Québec. ${ }^{26}$

Nous avons trouvé deux autres exemples de dispenses accordées et notées dans les registres de Beauport, le 21 novembre 1740 et le 30 juillet 1750 ; en 1740 , nous pouvons lire:

Dispense du 4e degré de parenté, donnée par Monsieur Hazeur, grand-penitencier, et vicaire général du diocèze. ${ }^{27}$

25 Ibid., 338.

26 Langevin, op. cit., 100.

27 Ibid., 171 . 
L'autre exemple est noté ainsi:

Dispense du troisième degré d'affinité, en datte du $25 \mathrm{e}$. des presens mois et an, signée $\dagger$ h. m. Episcopus Quebecensis, (Mgr Henri-Marie Dubreuil de Pontbriand) contre signée par Mre. Briand Chanoine Secretaire de Mond. Seigr. Evesque et scellée du sceau de ses armes. ${ }^{28}$

Dans les registres de la paroisse de Notre-Dame de Foy, datant de 1699 à 1760, nous n'avons découvert que deux cas de dispenses obtenues et notées; le premier est en date du 10 novembre 1699, il s'agit du mariage de Denis Mallet et de Geneviève Léonard; si nous passons les premières lignes nous lisons:

M'ayant mis entre les mains un mandement déclaratoire de Monseigneur de quebeq, par lequel il me permet de marier le dit Denys Mallet avec genviefve Leonard (...) nonobstant qu'elle soit cousine germaine de la defuncte femme (MarieMadeleine Jérémie, 1ère femme de Denys Mallet) et qu'elle ait tenu un de ses enfans sur les fonts de Bapteme, comme le dispensant de ces deux empechements, comme aussi de la publication des trois bans. ${ }^{29}$

L'autre mariage avec dispense date du 12 novembre 1753 et la notation se lit comme suit:

Apres avoir obtenu la dispense sy jointe pour le degré de consanguinité. ${ }^{30}$

La dispense dont il est fait mention n'est plus dans le registre; elle s'est perdue probablement, comme beaucoup d'autres papiers importants, que les ans, les incendies ou même des mains peu scrupuleuses ont fait disparaître!

En plus de ces dispenses demandées et obtenues, nous avons d'autres cas de mariages annulés après la connaissance d'empêchements dirimants. Le 30 août 1660 :

Une ordonnance de l'officialité de Mgr. de Petrée déclare nul, le mariage de Pierre Gadois et de Marie Pontenier célébré le 12 août 1657, à Québec, et la dite Marie Pontenier épouse à Montréal, le 3 novembre suivant, Pierre Martin. ${ }^{31}$

28 Ibid., 183s.

29 Registres de la Paroisse Notre-Dame de Foy, 1699.

30 Ibid., 1753.

31 Tanguay, op. cit., 41. 
Quelques années plus tard, le 24 octobre 1663, il est noté dans les Registres de Québec, dit l'abbé Tanguay, que:

Jacques Fournier, sieur de la Ville, qui avait épousé en 1657, aux Trois-Rivières, Marguerite Crevier, fille de Christophe Crevier, et dont le mariage avait été annulé, épousait à Québec Hélène Dufiguier. (...) D'après le registre du Conseil Souverain, le 3 nov. 1663, il apparaîtrait qu'il y avait empêchement dirimant. ${ }^{32}$

L'Église a établi certains empêchements aux mariages afin d'empêcher, ou du moins, de rendre plus difficiles, les mariages dont les suites pourraient être malheureuses; cependant elle accorde des dispenses lorsque les raisons sont jugées suffisantes; afin d'éviter les abus, elle fixe une taxe payable par ceux qui désirent obtenir une dispense, c'est ce qu'on appelle la componende. Avec les âges, le montant des componendes a varié; sous le Régime Français nous n'avons malheureusement trouvé aucun exemple nous indiquant le tarif à payer selon l'empêchement. Cependant, nous pouvons nous en faire une idée assez juste, en consultant les tarifs en vigueur en 1766 ; la dispense requise pour le troisième degré de consanguinité est fixée à 48 livres; pour le quatrième degré: 30 livres. ${ }^{33}$ Quelques années plus tard, en 1771, la componende pour le deuxième degré (entre cousins germains) est fixée à 500 livres: ${ }^{34}$ c'est une très forte somme, surtout pour le temps; ceci nous montre bien la ferme intention de l'Église d'éviter ces sortes de mariages.

Après avoir vu les notions dogmatiques sur le mariage, nous avons étudié ce qui est requis dans la préparation d'un mariage sous le Régime Français: la capacité des contractants en ce qui regarde leur âge, le consentement des parents, l'instruction des contractants et le choix du conjoint. Nous avons ensuite fait une brève étude sur les fiançailles solennelles, leur nature et les conséquences qu'elles entraînent. Puis l'annonce du mariage se fait solennellement au prône: ce sont les publications dont le but principal est de découvrir les empêchements qui pourraient s'oppo-

32 Ibid., 44.

33 RAPQ, (1947-1948) : 102.

34 Ibid., 109. 
ser à la célébration d'un mariage. Nous avons aussi étudié les principaux empêchements, soit dirimants soit prohibants; les premiers rendent le mariage invalide et nul tandis que les autres ne le rendent qu'illicite. Nous terminons done ici la préparation éloignée du mariage.

Il nous reste à étudier la célébration du mariage; en premier, nous étudierons la célébration religieuse, la plus importante, et nous verrons brièvement la célébration populaire. En premier lieu nous nous attarderons quelque peu sur la préparation immédiate du mariage, c'est-à-dire les circonstances de personnes, de lieu et de temps; puis nous passerons à l'étude des cérémonies du mariage, à la messe, à la tenue des registres, enfin nous étudierons quelques cas spéciaux qui se rattachent à notre travail.

\section{(à suivre)}

PAUL-ANDRÉ LECLERC, ptre, Licencié ès-lettres, Univ. Laval, Collège de Ste-Anne-de-la-Pocatière, P.Q. 